\title{
Changes in the pattern of DNA methylation associate with twin discordance in systemic lupus erythematosus
}

\author{
Biola M. Javierre, ${ }^{1}$ Agustin F. Fernandez, ${ }^{2}$ Julia Richter, ${ }^{3}$ Fatima Al-Shahrour, ${ }^{4,5,6}$ \\ J. Ignacio Martin-Subero, ${ }^{3}$ Javier Rodriguez-Ubreva, ${ }^{1}$ Maria Berdasco, ${ }^{2}$ \\ Mario F. Fraga, ${ }^{2}$ Terrance P. O'Hanlon, ${ }^{7}$ Lisa G. Rider, ${ }^{7}$ Filipe V. Jacinto, ${ }^{2}$ \\ F. Javier Lopez-Longo, ${ }^{8}$ Joaquin Dopazo, ${ }^{4,9}$ Marta Forn, ${ }^{10}$ Miguel A. Peinado, ${ }^{10}$ \\ Luis Carreño, ${ }^{8}$ Amr H. Sawalha, ${ }^{11,12,13}$ John B. Harley, ${ }^{11,12,13}$ Reiner Siebert, $^{3}$ \\ Manel Esteller, ${ }^{2}$ Frederick W. Miller, ${ }^{7}$ and Esteban Ballestar ${ }^{1,14}$
}

${ }^{1}$ Chromatin and Disease Group, Cancer Epigenetics and Biology Programme (PEBC), Bellvitge Biomedical Research Institute (IDIBELL), L'Hospitalet de Llobregat, Barcelona 08907, Spain; ${ }^{2}$ Cancer Epigenetics Group, Cancer Epigenetics and Biology Programme (PEBC), Bellvitge Biomedical Research Institute (IDIBELL), L'Hospitalet de Llobregat, Barcelona 08907, Spain; ${ }^{3}$ Institute of Human Genetics, University Hospital Schleswig-Holstein, Campus Kiel, Christian-Albrechts-University Kiel, Kiel D-24105, Germany; ${ }^{4}$ Bioinformatics Department, Centro de Investigación Príncipe Felipe, Valencia 46012, Spain; ${ }^{5}$ Broad Institute, Cambridge, Massachusetts 02142, USA; ${ }^{6}$ Hematology Division, Brigham and Women's Hospital, Harvard Medical School, Brookline, Massachusetts 02115, USA;

${ }^{7}$ Environmental Autoimmunity Group, National Institute of Environmental Health Sciences, NIH, HHS, Bethesda, Maryland 20892, USA; ${ }^{8}$ Division of Rheumatology, Gregorio Marañon Hospital, Madrid 28007, Spain; ${ }^{9}$ ISCIII Center for Biomedical Research on Rare Diseases, Valencia 46012, Spain; ${ }^{10}$ Institut de Medicina Predictiva i Personalitzada del Càncer (IMPPC), Badalona 08916, Spain;

${ }^{11}$ Arthritis and Immunology Program, Oklahoma Medical Research Foundation, Oklahoma City, Oklahoma 73104, USA;

${ }^{12}$ U.S. Department of Veterans Affairs Medical Center, Oklahoma City, Oklahoma 73104, USA; ${ }^{13}$ Department of Medicine, University of Oklahoma Health Sciences Center, Oklahoma City, Oklahoma 73104, USA

\begin{abstract}
Monozygotic (MZ) twins are partially concordant for most complex diseases, including autoimmune disorders. Whereas phenotypic concordance can be used to study heritability, discordance suggests the role of non-genetic factors. In autoimmune diseases, environmentally driven epigenetic changes are thought to contribute to their etiology. Here we report the first high-throughput and candidate sequence analyses of DNA methylation to investigate discordance for autoimmune disease in twins. We used a cohort of $M Z$ twins discordant for three diseases whose clinical signs often overlap: systemic lupus erythematosus (SLE), rheumatoid arthritis, and dermatomyositis. Only MZ twins discordant for SLE featured widespread changes in the DNA methylation status of a significant number of genes. Gene ontology analysis revealed enrichment in categories associated with immune function. Individual analysis confirmed the existence of DNA methylation and expression changes in genes relevant to SLE pathogenesis. These changes occurred in parallel with a global decrease in the 5-methylcytosine content that was concomitantly accompanied with changes in DNA methylation and expression levels of ribosomal RNA genes, although no changes in repetitive sequences were found. Our findings not only identify potentially relevant DNA methylation markers for the clinical characterization of SLE patients but also support the notion that epigenetic changes may be critical in the clinical manifestations of autoimmune disease.
\end{abstract}

[Supplemental material is available online at http://www.genome.org. The sequence data from this study have been submitted to the NCBI Gene Expression Omnibus (http://www.ncbi.nlm.nih.gov/geo) under accession no. GSE19033.]

Human monozygotic (MZ) twins exhibit variable degrees of concordance for complex diseases, such as cancer, cardiovascular diseases, or autoimmune disorders. Whereas concordance rates close to $100 \%$ in identical twins apply to coinheritance of mutant genes that are dominant and highly penetrant, most diseases or traits show a concordance in identical twins in the broad range of 5\%-75\% (Nance 1978). Most of the twin-based studies have focused on the concor-

\footnotetext{
${ }^{14}$ Corresponding author.

E-mail eballestar@idibell.org; fax 34-93-2607219.

Article published online before print. Article and publication date are at http://www.genome.org/cgi/doi/10.1101/gr.100289.109.
}

dance between siblings that has led to the identification of traitspecific genes (Hrubec and Robinette 1984), while less attention has been paid to the degree of discordance, which suggests the participation of factors other than pure genetic changes. Recently, interest has shifted toward exploring the molecular mechanisms involved in determining phenotypic differences. The increasing recognition of the influence of epigenetics in phenotypic outcomes continues to open up new lines of research involving twin studies. DNA methylation and histone modifications, the major sources of epigenetic information, regulate gene expression levels and provide an alternative mechanism for modulating gene function to those arising from genetic changes (Esteller 2008). Interestingly, epigenetic changes are 
highly dependent on environmental factors, and recent data support the notion that diet and other external influences can alter the epigenetic status of genes (Mann et al. 2004; Heijmans et al. 2008).

In the case of autoimmune disorders, twin studies have provided evidence of the existence of a genetic component and have been useful for identifying susceptibility genes. One of the best studied autoimmune diseases is systemic lupus erythematosus (SLE), an archetypical systemic, autoimmune inflammatory disease characterized by the production of autoantibodies to multiple nuclear antigens. Well-established risk factors include alleles in the major histocompatibility complex region, IRF5, STAT4, BLK, BANK1, PDCD1, MECP2, and TNFSF4, among others (Graham et al. 2007; International Consortium for Systemic Lupus Erythematosus Genetics 2008; Moser et al. 2009). Many SLE susceptibility genes belong to key pathways that are common to those associated with other autoimmune disorders. In fact, not only genetic factors but also clinical signs and symptoms of SLE overlap with those of other autoimmune diseases such as rheumatoid arthritis (RA) or dermatomyositis (DM) (Encinas and Kuchroo 2000) (a summary of clinical features of these three diseases is presented in Supplemental Table 1).

In addition to twin discordance, additional evidence highlights the relevance of epigenetic events in the etiology of SLE and other autoimmune diseases. Lymphocytes from patients with SLE and RA exhibit globally hypomethylated DNA (Richardson et al. 1990; Corvetta et al. 1991). In parallel, DNA methylation analysis of several candidate genes in lymphocytes from SLE individuals has identified promoter demethylation that may contribute to their aberrant overexpression. These changes occur in PRF1 (Kaplan et al. 2004), CD70 (Lu et al. 2005), ITGAL (Lu et al. 2002), and the $X$ chromosome gene CD4OLG (Lu et al. 2007) promoters, and are known to be associated with the development of lupus. Additional evidence of the role of DNA methylation changes in the development of SLE comes from studies of DNA demethylating drugs. For instance, $\mathrm{CD} 4^{+} \mathrm{T}$-cells become autoreactive following treatment with 5-azacytidine (Richardson 1986), which causes genome-wide hypomethylation and aberrant overexpression of many genes.

Direct comparison of identical twins is an excellent experimental approach for testing environmental epigenetics, because DNA sequence differences, including single-nucleotide polymorphisms, which would be abundant in singleton-based studies, do not interfere in this analysis. Recent studies with twins (Fraga et al. 2005; Kaminsky et al. 2009) have demonstrated the existence of genome-wide epigenetic differences that potentially could explain differences in phenotype. Here, we used a collection of identical twins discordant for SLE and two other related systemic autoimmune diseases to perform the first highthroughput analysis of DNA methylation changes. Interestingly, only SLE samples exhibited significant changes in DNA methylation status at both the global and sequence-specific levels in comparison with their healthy, discordant twins and compared with unrelated matched normal controls. Our study allowed us to identify differential methylation between SLE and healthy twins in a set of genes with biological functions relevant to SLE pathogenesis. We also found a global decrease in global 5-methylcytosine $(5 \mathrm{mC})$ content when comparing SLE twins with their corresponding healthy twins. We also observed a change in DNA methylation status of the CpG-rich region of the ribosomal DNA repeat, which contains the transcribed $18 \mathrm{~S}$ and $28 \mathrm{~S}$ genes. Our twin study provides the first evidence that large changes in the DNA methylation profile may be associated with the differential onset of the disease in twins discordant for SLE, suggesting that there is an association with environmental effects. In addition, our study also yields a list of epigenetically deregulated DNA sequences in SLE, which may potentially lead to a better understanding of its pathogenesis.

\section{Results}

\section{DNA methylation profiling reveals differences in $\mathrm{MZ}$ twins discordant for SLE}

To investigate the existence of gene-specific DNA methylation changes associated with the development of the disease in MZ twins discordant for SLE, RA, and DM, we used a high-throughput approach. Samples had been collected when the affected twins showed signs of disease activity. Specifically, we performed DNA methylation profiling using universal bead arrays (Bibikova et al. 2006) with 60 samples corresponding to DNA extracted from white blood cells (WBCs) of $30 \mathrm{MZ}$ twins discordant for SLE, RA, and DM (five twin pairs of each disease), and 30 unrelated normal controls matching by pairs in age, gender, and race with the MZ twin pairs studied. This bead array interrogates the DNA methylation status of 807 CpG-containing promoters of genes classified in various groups such as those pertaining to cell cycle control, differentiation, apoptosis, imprinting, and so on. This approach enabled us to obtain highly reproducible DNA methylation profiles. Scatterplots comparing the average DNA methylation patterns of the five MZ twins discordant for each RA and DM illustrate the absence of differences (Fig. 1A), indicating that, at least for the 807 promoters represented in this array, there had been no significant changes in DNA methylation status in these two diseases. In contrast, when comparing the five SLE pairs of discordant MZ twins, we found a common set of genes exhibiting differential DNA methylation in SLE twins compared with their healthy twins. Statistical analysis of the combined data from the five MZ twins discordant for SLE showed that 49 genes had significant differences in DNA methylation $(\beta>0.10, P$-value $<0.05$, Student's $t$-test) between SLE and healthy MZ twins (Fig. 1B,D; Supplemental Table 2). The comparisons between MZ twins, which are genetically identical, avoid the effects of any potential interference that SNPs might cause in this sort of analysis. The specificity of the differences observed is highlighted by the fact that those changes corresponding to the average of five pairs of MZ twins discordant for SLE were almost identical to the pattern obtained for each of the individual MZ twin pairs (Fig. 1, cf. left panels of B and C). Correlation between cell subfractions (as obtained by automated complete blood counts) and methylation data of these 49 genes confirmed that the methylation differences observed in WBC samples were not due to cell subfraction changes. The fact that the level of difference is moderate is not unexpected, given the heterogeneous nature of human subjects and lupus syndromes as well as the complex nature of the biological samples used in this study. It is of particular interest that this set of 49 genes, which are differentially methylated in SLE and healthy MZ twins, showed no significant methylation changes when compared with the DNA methylation profiles of the five pairs of unrelated normal controls matched by age, gender, and race (see right panels of Fig. 1B,C), suggesting that the aforementioned DNA methylation differences are specific to the different phenotypes of SLE and healthy twins.

We then set out to determine whether the 49 differentially methylated genes could be involved in biological functions relevant to the pathogenesis of SLE. As a first step, we performed Gene Ontology analysis to test whether some molecular functions, 
A

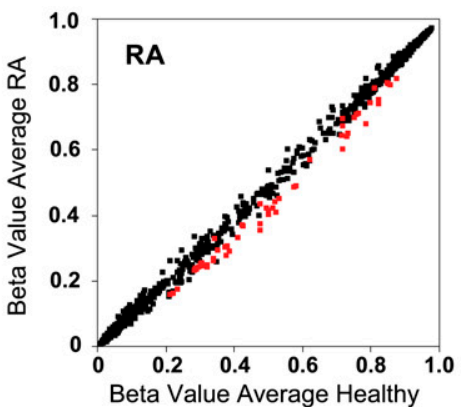

B

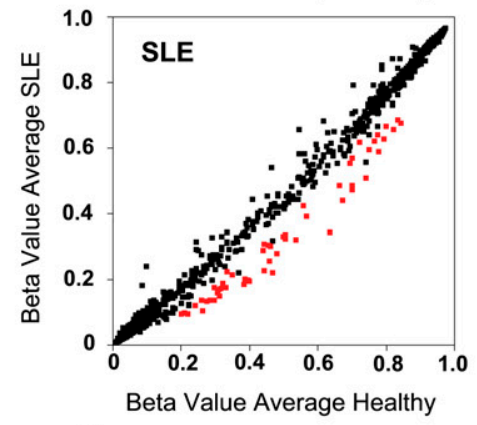

C

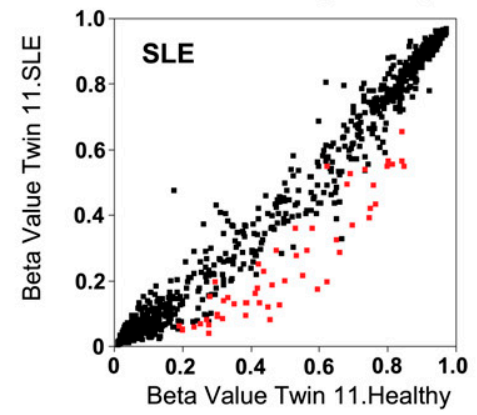

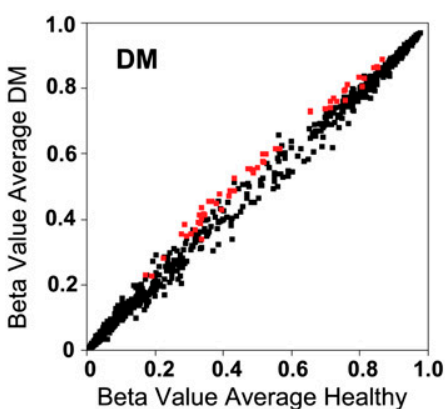

D MATChed CONTROLS HEALTHY TWINS SLE TWINS
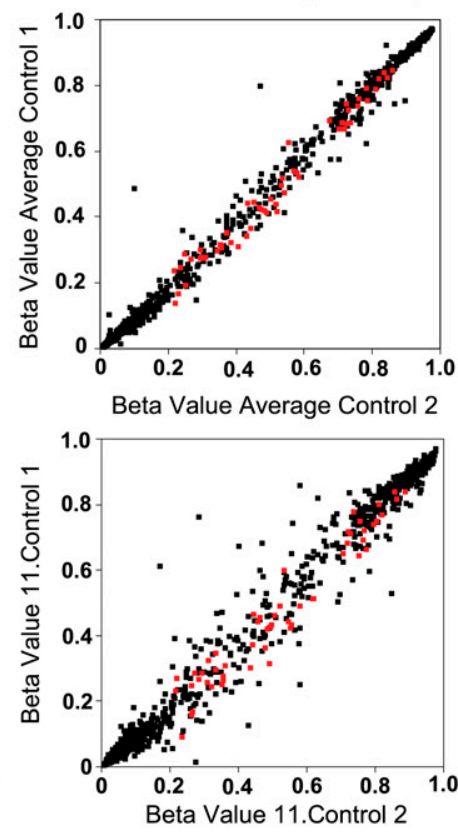

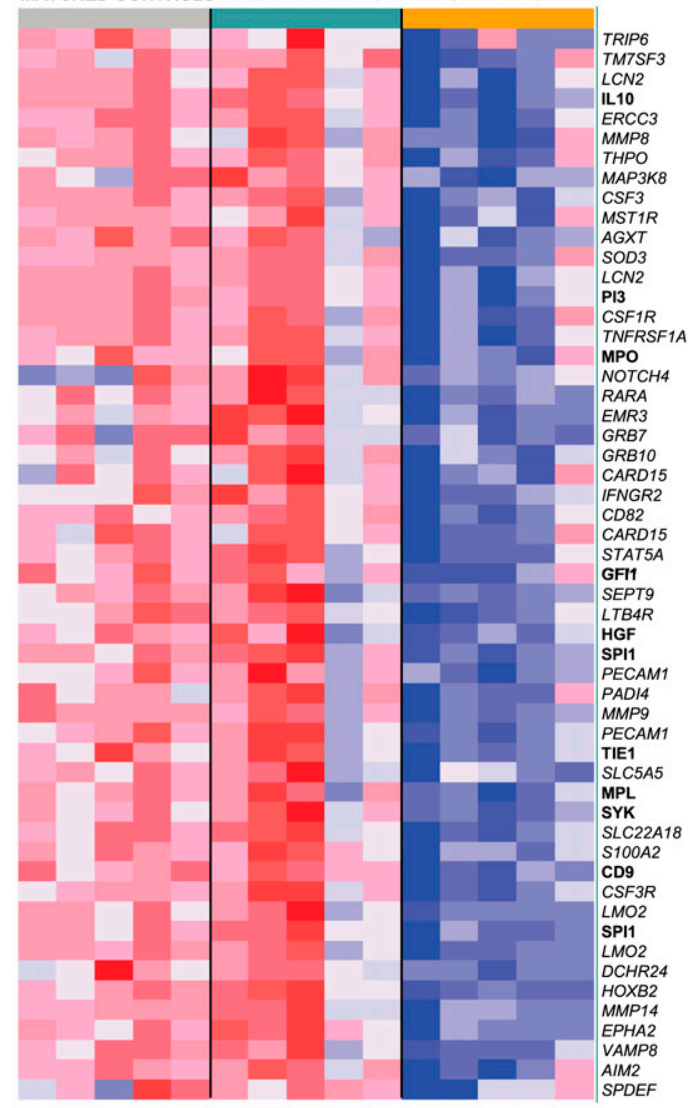

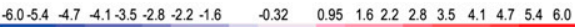

Figure 1. Representative scatterplots showing methylation profiles of twins with the three autoimmune diseases studied (SLE, RA, and DM) compared with their respective healthy twins. (A) Scatterplots corresponding to the average data from five MZ twin pairs discordant for RA (left) and DM (right). (Red) Genes with significant differences in averaged results from five SLE-discordant MZ twin pairs (see below). (B) (Left) Scatterplot corresponding to the averaged results from five SLE-discordant MZ twin pairs. (Right) Comparison of the averaged data of five pairs of unrelated controls matched by race, age, and gender with samples from the left panel. (Red) Significant genes $(\beta>0.10, P<0.05)$. (C) (Left) A comparison of a single SLE-discordant MZ twin pair. (Red) Genes with significant differences in averaged results from five MZ twin pairs (see above). (D) Heat map including the data for the 49 genes (54 probes) showing differential methylation between SLE affected and healthy MZ twin pairs (five samples were included). Five additional age-, gender-, and race-healthy controls for each of the five MZ twin pairs are also included. Data from the Illumina array have been normalized as follows: $x i=(x i-$ row.mean $[i]) /(0.333 \times$ row.sd[i]). A scale is shown at the bottom, whereby positive (red) and negative (blue) values correspond, respectively, to a higher and a lower methylation status than average.

biological processes, or the cellular components were significantly associated with the genes with the greatest difference in DNA methylation status between the SLE discordant MZ twins. Interestingly, we observed significantly enriched functional processes that are potentially relevant in autoimmune inflammatory diseases, including the following categories: defense response (GO:0006952), cell activation (GO:0001775), immune response (GO:0006955), cell proliferation (GO:0008283), or cytokine production (GO:0001816) (see Supplemental Table 3). This range of associated functions suggests potential cell types and biological pathways affected by these aberrant DNA methylation changes.

To confirm that these differences in DNA methylation between SLE discordant MZ twins were robust, we carried out bisulfite genomic sequencing of multiple clones within promoter regions of selected genes comprising the fragment corresponding to the oligonucleotide probe represented in the methylation array. This experiment allowed us not only to confirm the existence of DNA methylation changes in these genes, but also to analyze the regions neighboring the $\mathrm{CpG}$ sites included in the bead array. We chose the following genes, given the level of difference in methylation revealed by the methylation arrays, their functional relevance, or existing literature: IFNGR2, MMP14, LCN2, CSF3R, PECAM1, CD9, AIM2, and PDX1. To confirm the previous analysis, we chose two MZ twin pairs discordant for SLE, two MZ twin pairs concordant for SLE, two healthy MZ twin pairs, and two unrelated healthy race-, gender-, and age-matched controls (several examples are shown in Supplemental Fig. 1). This analysis confirmed the existence of DNA methylation differences between SLE-discordant MZ twins. In addition, it showed that the levels of methylation for these genes in the SLE sibling are comparable to those observed in each of the individuals of concordant SLE twin pairs. To gather further evidence from a wider selection of samples, we pyrosequenced a set of 17 pairs of siblings discordant for SLE. In addition to the first set of five pairs of MZ twins, we included one additional pair of MZ twins, four dizygotic (DZ) twin pairs discordant for SLE, and seven pairs of non-twin siblings within $4 \mathrm{yr}$ of age discordant for

\section{Genome Research} www.genome.org 
SLE. We observed a remarkable decrease in the DNA methylation levels of SLE subjects with respect to their corresponding healthy siblings for the majority of genes tested (Fig. 2), regardless of whether the subject pairs were MZ twins, DZ twins, or non-twin siblings discordant for SLE. A Student's $t$-test revealed that seven of the eight genes (IFNGR2, MMP14, LCN2, CSF3R, PECAM1, CD9, and AIM2, but not $P D X 1)$ exhibited a significant reduction $(P<0.05)$ in their DNA methylation levels between SLE and corresponding healthy
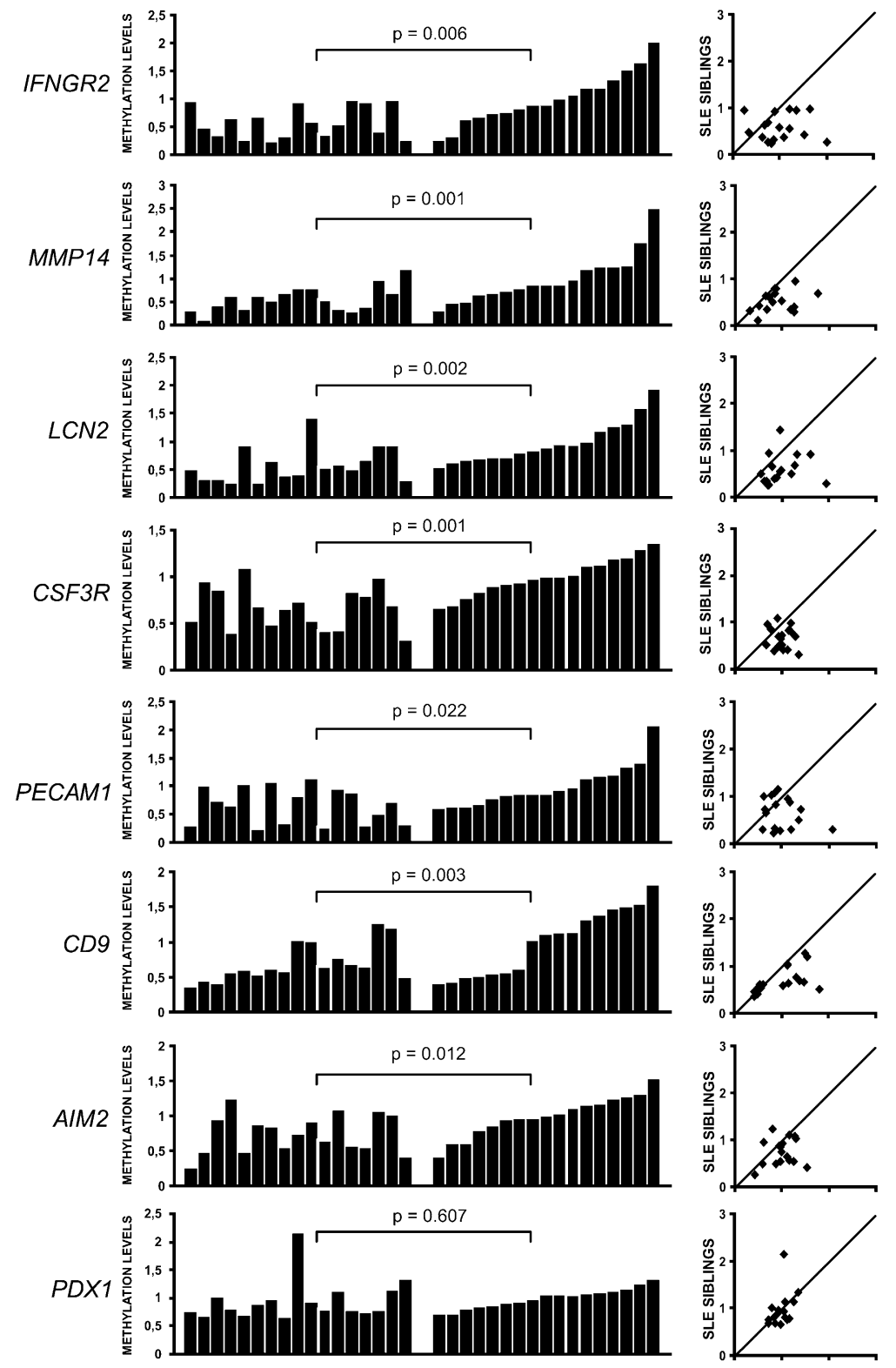

SLE SIBLINGS

HEALTHY SIBLINGS
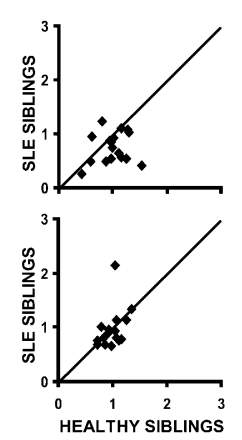

Figure 2. A comparison of the DNA methylation levels of eight genes in paired samples discordant for SLE. Bisulfite pyrosequencing analysis was performed on the genes selected from experiments with methylation arrays. Methylation levels were normalized by dividing the percentage of methylation for each particular gene by the average percentage of methylation in the entire population (17 SLE discordant sibling pairs: six MZ twins, four DZ twins, and seven disease-discordant sibling pairs). Control samples are ordered right to left by decreasing level of methylation. SLE samples are on the left side of each graph, and the same order is maintained for their respective healthy twins or siblings. Each bar graph is accompanied by a scatterplot representing the normalized DNA methylation level of each gene for the control samples relative to their equivalent SLE sample. siblings, indicating that these changes in the DNA methylation profile are robust. We examined by Pearson test the relationship between DNA methylation changes in these genes and clinical data fhe SLE individuals (activity and damage indexes), age and race. No significant correlation was observed, although a larger ulation size might be necessary for such analysis

DNA methylation changes within the promoter region of dulate their expression levels. To investigate this possibility, we performed quantitative RT-PCR to compare the expression levels of these seven genes for the five MZ twin pairs discordant for SLE. We found that for most of the genes tested (IFGNR2, MMP14, LCN2, CSF3R, and AIM2), the observed reduction in their DNA methylation status was correlated with increased expression levels when comparing SLE individuals versus their corresponding healthy siblings (Supplemental Fig. 2A).

Global loss of 5-methylcytosine in SLE twins is not associated with changes at Alu, Satellite2, D4Z4, and NBL2 repeats

Previous findings from other laboratories have shown decreased global DNA methylation levels to be associated with the development of SLE and RA (Richardson et al. 1990; Corvetta et al. 1991). In order to establish the existence of global DNA methylation changes in our set of samples, we measured the total content of $5 \mathrm{mC}$ in the same 17 pairs of siblings discordant for SLE. We observed a robust decrease in the $5 \mathrm{mC}$ content of SLE individuals compared with their corresponding healthy twins or siblings (Fig. $3 \mathrm{~A}$ ) (Student's $t$-test $=-2.589, P=0.021$ ). To determine whether this change in DNA methylation content was associated with a decrease in the levels of DNA methyltransferases, we performed quantitative RT-PCR of DNMT1 and DNMT3B and found significant reductions in the mRNA levels of DNMTs, specifically of $\sim 35 \%$ for DNMT1 and 20\% for DNMT3B (Supplemental Fig. 2B).

A global decrease in the $5 \mathrm{mC}$ content cannot exclusively be associated with changes in the DNA methylation status of genes, since these constitute a very small proportion of the total genomic CpG content. In humans, most of the methylated cytosines are found in CpG-rich sequences within tandem and interspersed repeats that constitute up to $45 \%$ of the human genome, and Alu repeats are the most common family of such repeats. We used genome-wide amplification of unmethylated DNA Alu repeats (AUMA) (Rodriguez et al. 2008) to 
A

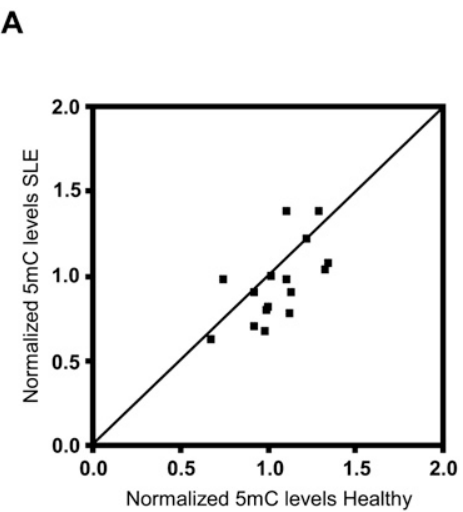

B

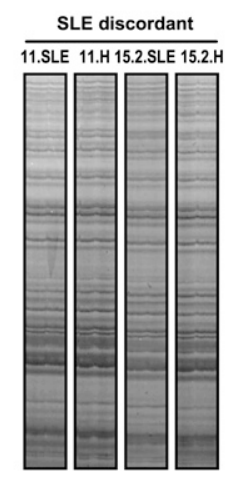

C

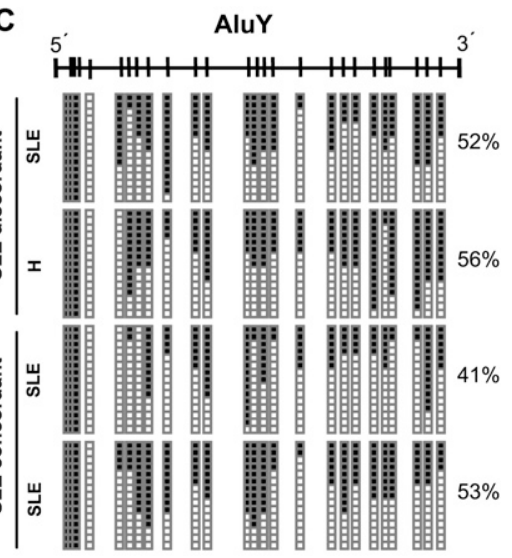

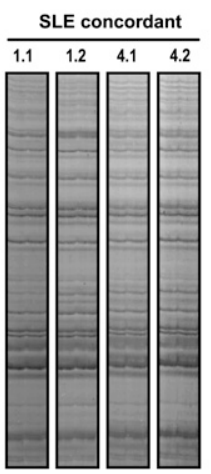

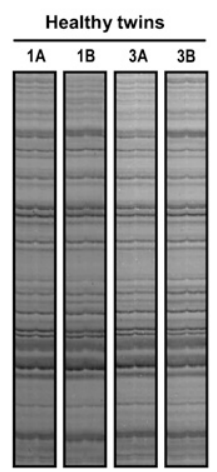

Figure 3. (A) Scatterplot comparing total $5 \mathrm{mC}$ content in SLE siblings and their matching healthy siblings. Data are normalized with respect to the average $5 \mathrm{mC}$ methylation content in the entire population. (B) Band patterning corresponding to the analysis of unmethylated/methylated Alu repeats. Two SLE discordant MZ twin pairs, two SLE concordant MZ twin pairs, and two concordant pairs of healthy MZ twins are shown. To show the sensitivity toward DNA methylation changes, a WBC sample is compared with the same sample following limited treatment with SssI DNA methyltransferase. (C) Individual bisulfite sequencing of consensus Alu repeat. Fifteen clones are shown. (Black squares) Methylated CpG sites; (white squares) non-methylated CpG sites. One pair of SLE discordant MZ twins and one pair of SLE concordant MZ twins are shown.

compare the genomic-scale levels of unmethylated and methylated Alu elements of a selection of MZ twins discordant for SLE, MZ twins concordant for SLE, and unrelated healthy MZ twins. No significant differences were observed (Fig. 3B). The absence of significant changes was confirmed by performing bisulfite genomic sequencing of a consensus Alu repeat (Fig. 3C), although small changes in two specific CpGs within the Alu repeat sequence were observed. Several additional repetitive sequences have been shown to undergo DNA methylation changes in cancer and to exhibit a variety of genetic disorders. We decided to analyze the DNA methylation profile of some of these sequences, including satellite2 repeats, NBL2 and D4Z4 non-satellite subtelomeric repeats, and LINE-1 repeats. Comparison of the DNA methylation profiles in the five original MZ twin pairs discordant for SLE that had previously been shown to have differences in the global content of $5 \mathrm{mC}$ showed no significant differences in the DNA methylation status of any of these repeat sequences (two examples are illustrated in Supplemental Fig. 3).

\section{SLE discordant MZ twins are differentially methylated at ribosomal RNA genes}

Ribosomal genes are present in significant copy numbers (150300) in mammalian genomes. Interestingly, aberrant changes in

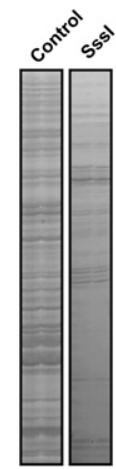

the DNA methylation status of ribosomal genes, particularly in the transcribed region containing the $28 \mathrm{~S}, 18 \mathrm{~S}$, and $5.8 \mathrm{~S}$ that resembles a large CpG island, have been associated with various disorders. For instance, in $\alpha$-thalassemia/mental retardation syndrome X-linked (ATRX), hypomethylation of the ribosomal genes at chromosomes $13,14,15,21$, and 22 is known to be associated with mutations in ATRX, a chromatin remodeling factor (Gibbons et al. 2000). Also, genetic manipulation of DNMT levels reduces the DNA methylation levels of ribosomal genes (Espada et al. 2007). We therefore performed bisulfite genomic sequencing for the five original pairs of MZ twins discordant for SLE on three regions of the ribosomal RNA gene cluster: the ribosomal gene promoter, $18 \mathrm{~S}$, and $28 \mathrm{~S}$. Although no significant differences in DNA methylation were observed in the promoter region, we found a significant decrease in the $18 \mathrm{~S}$ and $28 \mathrm{~S}$ segments (Fig. 4A) for the SLE sibling of each twin pair. Healthy siblings of SLE-discordant twin pairs had similar DNA methylation levels to those of healthy twin pairs and other unrelated age-, gender-, and racematched normal controls (Fig. 4A). In contrast, individuals from twin pairs concordant for SLE exhibited low levels of methylation for the $18 \mathrm{~S}$ and $28 \mathrm{~S}$ sequences, similar to those of the SLE sibling of discordant twin pairs.

We then performed bisulfite sequencing to determine the DNA methylation status of all 17 pairs of siblings discordant for SLE and seven pairs of SLE-concordant MZ twins. Our results confirmed significantly decreased methylation of the $18 \mathrm{~S}$ and $28 \mathrm{~S}$ ribosomal genes in all SLE samples relative to their respective healthy siblings, as revealed by a Student's $t$-test $(P<0.05)$ (Fig. 4B,C; Supplemental Table 4). Three comparisons were tested: (1) SLE siblings from discordant twin pairs with their respective healthy siblings, (2) SLE siblings from discordant twin pairs with SLE-concordant MZ twins, and (3) healthy siblings from discordant twin pairs with SLE-concordant MZ twins. Unpaired twotailed $t$-tests revealed that $18 \mathrm{~S}$ and $28 \mathrm{~S}$ are hypomethylated in SLE siblings with respect to their corresponding healthy siblings and that they exhibit similar values to individuals from SLE-concordant MZ twin pairs (Fig. 4B,C).

It is likely that changes in the DNA methylation status of ribosomal genes, even when they occur at regions downstream from the transcription start site, may affect their transcriptional rate. Thus, we assessed the effects on rRNA expression at two levelsfirst, by looking at the expression levels of the full-length precursor RNA that is transcribed; and, second, by examining the mature 18S RNA. When we compared the expression levels of the precursor RNA and the 18S RNA by quantitative RT-PCR in the six pairs of MZ twins discordant for SLE, both transcripts showed higher levels in all the SLE twins compared with their corresponding healthy twins. This suggests that changes in the DNA

\section{Genome Research} www.genome.org 


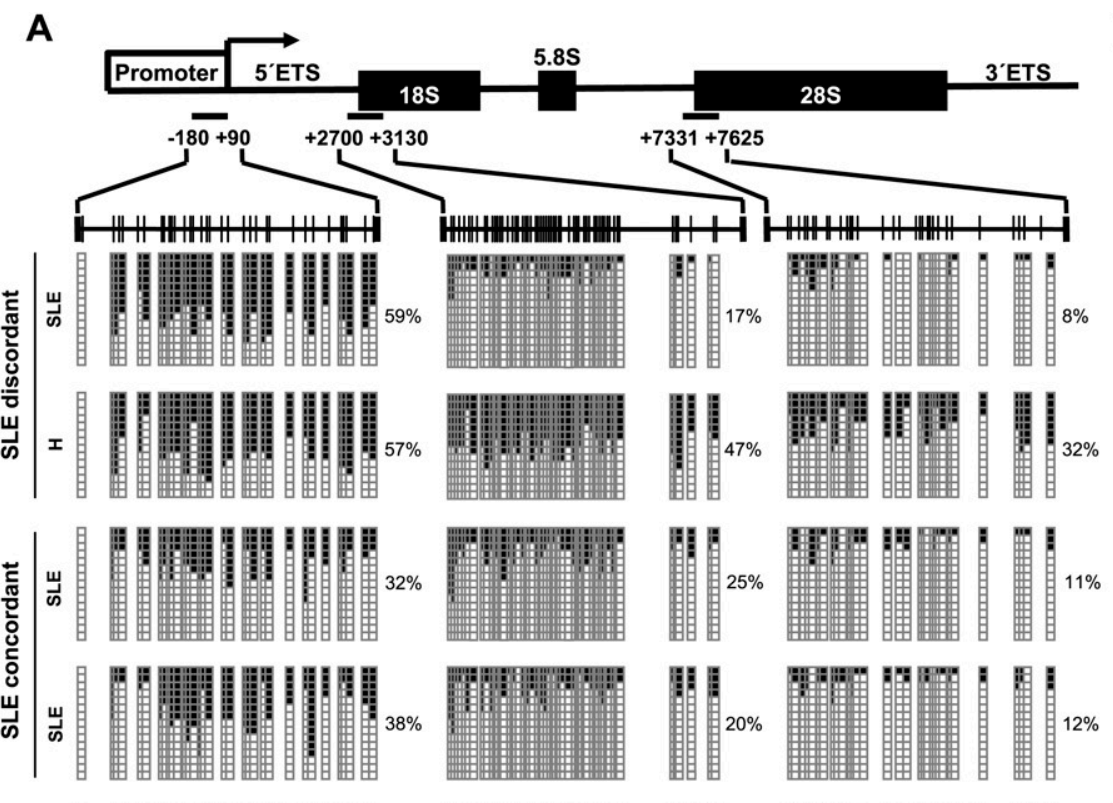

B
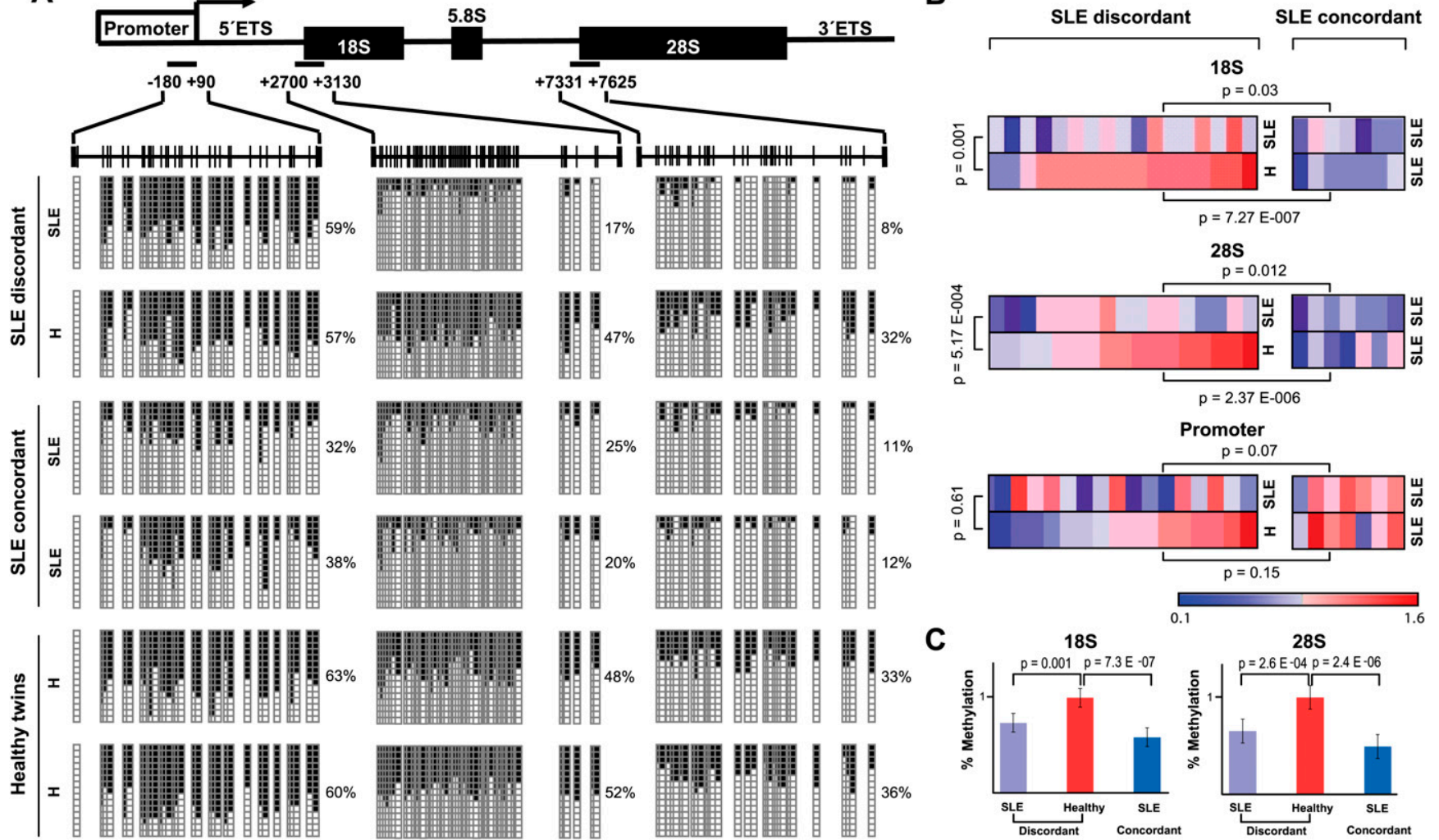

Figure 4. DNA methylation changes in ribosomal genes in paired samples discordant for SLE. (A) Schematic representation of the rRNA gene depicting three regions in the gene repeat: the proximal promoter, including the transcription start site, and the initial sections of the $18 \mathrm{~S}$ and $28 \mathrm{~S}$ regions, were subjected to bisulfite genomic sequencing. Fifteen clones are shown. (Black squares) Methylated CpG sites; (white squares) non-methylated CpG sites. One SLE discordant pair of MZ twins, one SLE concordant pair of MZ twins, and one concordant pair of healthy MZ twins are shown. (B) Heat map including the normalized methylation data for 17 pairs of SLE-discordant samples and seven pairs of SLE-concordant samples. Data are normalized with respect to the average value of healthy siblings from discordant twin pairs, and a color scale is established between no-methylation (blue) and methylation (red) values near the average of normal samples. Three comparisons are made: SLE siblings from discordant pairs versus SLE twins from concordant pairs (upper $P$-value); healthy siblings from discordant pairs versus SLE twins from concordant pairs (lower $P$-value); SLE siblings from discordant pairs versus their matching healthy twins ( $P$-value at left). (C) Bar graph showing the average percentage of DNA methylation for the $18 \mathrm{~S}$ and $28 \mathrm{~S}$ sequences of SLE siblings from discordant twin pairs, healthy siblings from discordant twin pairs, and SLE individuals from concordant twin pairs.

methylation profile were directly associated with the increased transcript levels (Supplemental Fig. 2B). To investigate whether these changes in the ribosomal RNA genes occurred in conjunction with changes in the DNA methylation status and/or expression of ribosomal proteins, for which auto-antibodies have been reported to be present in SLE patients, we performed bisulfite genomic sequencing of the promoter $\mathrm{CpG}$ island and quantitative RT-PCR for RPLPO, RPLP1, and RPLP2 of the six pairs of MZ twins discordant for SLE. Neither methylation changes nor expression changes were found when comparing SLE and healthy twins. Further studies will be necessary to determine the functional relevance of changes at the DNA methylation and expression levels of ribosomal RNA.

\section{Discussion}

Many complementary lines of evidence suggest that epigenetic factors may play a key role in the pathogenesis of autoimmune diseases. Interactions between environmental and genetic factors have been proposed to explain why certain individuals develop autoimmune disorders while others do not (Gourley and Miller 2007), and provide an explanation for the high discordance rates for autoimmune diseases in MZ twins. On the other hand, several
SLE susceptibility genes, like STAT4 or MECP2, have mechanistic connections with DNA methylation. The existence of functionally relevant polymorphisms in genes that can influence DNA methylation status also highlights the need to investigate epigenetic changes involved in this disease.

In this context, our study constitutes an effort to understand the relevance of DNA methylation alterations in the context of autoimmune disease. Here we report the first high-throughput analysis of DNA methylation changes in autoimmune disease. In addition, we take advantage of the use of MZ twins to screen DNA methylation changes in the search for evidence that DNA methylation changes arise from a variety of environmental effects or are determined by specific genetic features of SLE individuals.

The rationale for the comparison of three autoimmune diseases was based on certain shared clinical signs and symptoms, immune responses, and common genetic risk factors. Despite these shared features, however, our data do not suggest the existence of common targets of epigenetic deregulation in these three disorders. Nonetheless, the possibility that they share epigenetic targets cannot be ruled out, and further studies involving the epigenomic analysis of a wider range of genes and perhaps additional target tissues are needed. 
Our results do indicate the existence of a decrease in the DNA methylation levels of several genes. A few studies have demonstrated that lymphocyte differentiation and activation are associated with loss of DNA methylation at key CpGs of promoters of genes such as interferon gamma and various cytokine genes (Murayama et al. 2006; Kim et al. 2007). Recent data indicate that active demethylation in vertebrates takes places through the cooperation of a deaminase, a mismatch-specific thymine glycosylase, and the nonenzymatic factor Gadd45 (Rai et al. 2009). Interestingly, Gadd45a-deficient mice develop a lupus-like syndrome (Salvador et al. 2002). Given the nature of our biological samples, we cannot draw any conclusion about the functional meaning of these methylation changes. However, we cannot discard that differences in activation of the subpopulations, which have been shown to be associated with DNA demethylation processes (Murayama et al. 2006; Kim et al. 2007), may participate in those changes. Further studies using fractionated cell populations will help to associate specific DNA methylation and gene expression changes with particular functions. In addition, the use of noninflammatory cell types such as hepatocytes or myocytes or more accessible cells such as buccal mucosa or skin will be useful for future studies. At any rate, WBCs are commonly used in the search for novel SLE targets and have provided specific signatures associated with SLE pathogenesis (Bennett et al. 2003; Chaussabel et al. 2008).

The importance of the genes identified in this study in the context of SLE and other autoimmune diseases becomes more evident upon performance of Gene Ontology analysis and review of existing literature. First, Gene Ontology analysis of the set of 49 differentially methylated genes reveals a significant enrichment in functional processes that are potentially relevant in autoimmune inflammatory diseases, such as immune response, cell activation, or response to external stimuli. Second, among the genes that we have demonstrated to feature lower methylation levels in SLE individuals than their healthy siblings, we have found several notable markers, some of which had been previously referenced in the context of SLE pathogenesis. For instance, interferon gamma receptor 2 (IFGNR2) has been shown to be associated with lymphadenopathy, splenomegaly, and kidney damage in the SLE mouse model MRL-Fas ${ }^{l p r}$ (Schwarting et al. 1998). Recent studies of interferon activity and lupus have suggested that interferon-stimulated genes, such as AIM2, participate in the development of SLE (Choubey and Panchanathan 2008). Dysregulation of colonystimulating factor 3 receptor (CSF3R) had previously been implicated in the defective expression of CD80 and the subsequent dysfunction of the antigen-presenting cells in SLE (Funauchi et al. 2002). Matrix metalloproteinases, such as MMP14, contribute to tissue destruction, regeneration, inflammation, and apoptosis, and several of them are up-regulated by a range of injuries in the skin and are thought to be involved in some of the organ manifestations of SLE. LCN2 is a small protein involved in iron transport and a common marker for lupus nephritis (Schwartz et al. 2007). LCN2 overexpression has recently been associated with renal dysfunction and is involved in apoptosis induction (Pitashny et al. 2007). Finally, CD9 has also been shown to be highly expressed in B-cells from lupus-prone mice (Duan et al. 2007), although its potential role in pathogenesis remains to be elucidated.

In parallel, a robust decrease in the global content of DNA methylation as well as a reduction of DNMT1 and DNMT3B mRNA levels were observed. Interestingly, mouse knockout models for Dnmt1 and Dnmt3b have also shown defects in global DNA methylation (Yung et al. 2001; Dodge et al. 2005). Heterozygous Dnmt1 null mutation mice developed autoimmunity, although surprisingly, they displayed increased DNA methylation levels (Yung et al. 2001). In humans, decreased levels of DNMT1 mRNA had previously been shown for T-cells isolated from SLE patients (Deng et al. 2001). Changes in DNMT1 and DNMT3b levels suggest a potential direct implication in global reduction of $5 \mathrm{mC}$. At any rate, DNA methylation analysis of a variety of repetitive sequences, including, Alu, LINE-1, D4Z4, NBL2, and Satellite 2 repeats, did not show significant differences in methylation between SLE and healthy individuals. This finding differs from those in cancer and other diseases, in which a global decrease in the content of $5 \mathrm{mC}$ is accompanied by the loss of methylation in different subsets of repetitive sequences. For instance, demethylation of Alu elements occurs in aging and cancer processes and has been associated with gene reactivation and genomic instability. In immunodeficiency, centromeric instability and facial anomalies (ICF) syndrome, satellite repeats, and non-satellite repeats like D4Z4 and NBL2 are hypomethylated (Kondo et al. 2000). In other cases, such as in facioscapulohumeral muscular dystrophy (FSHD), DNA methylation changes are even more localized and exclusively affect D4Z4 repeats (van Overveld et al. 2003). LINE-1 elements are other repetitive elements that are sensitive to DNA methylation changes in cancer or other disorders. We found instead a lower methylation content in ribosomal sequences. This finding may be of particular significance, since hypomethylation and overexpression of ribosomal RNA genes may be associated with an increased assembly of ribosomal particles that might stimulate the generation of autoantibodies against them. In fact, the presence of auto-antibodies against ribosomal RNA and proteins in SLE patients has been well documented over the last three decades. It is unlikely that decrease in DNA methylation at rRNA genes could solely account for global changes in the $5 \mathrm{mC}$ content, and perhaps other repetitive elements not assessed may also contribute.

Our investigation demonstrates the utility of twin studies for identifying epigenetic changes in disease. Given that MZ twins share their entire genotype (and potentially specific variants in SLE susceptibility genes), our results reinforce the notion that, for a particular genetic background, environmental factors can modulate the onset of SLE. However, at the current stage, we cannot conclude whether epigenetic changes are cause or consequence; in other words, whether environmental factors caused the epigenetic changes in these cell populations, which, in turn, cause the SLE or whether environmental factors cause the SLE by some unknown mechanism, which then is accompanied by inflammatory and immune responses that are secondary to the SLE.

A potential constraint on the use of this approach arises from the fact that many MZ monochorionic twins exchange blood through shared vascular communication. Therefore, if any DNA methylation changes occur in the blood of one of the twins before birth, it is common for both of them to show it. Only the sibling of the pair in whom the change has originally occurred might present the phenotype, because additional cells of the body could be affected. Therefore, MZ twins are a good model system for diseases that are not congenital, like autoimmune diseases, and that start to develop after birth, when there is no blood sharing. Our data do not allow us to conclude the timing of the DNA methylation changes observed; however, it is likely that they are associated with the onset of the disease.

Our recent study revealed the existence of epigenetic differences between MZ twins and demonstrated how these differences become more pronounced with age, supporting the notion that "epigenetic drift" plays a role in divergence of MZ phenotypes (Fraga et al. 2005). Data obtained from MZ and DZ twins provide

\section{Genome Research}

www.genome.org 
further evidence that differences in epigenomes can explain phenotypic differences (Kaminsky et al. 2009). Thus, the present report, in which the discordance between twins is used to identify epigenetic targets, highlights the potential of this strategy for learning about the contribution of epigenetics to autoimmune and other diseases.

\section{Methods}

\section{Samples}

All subjects, or in the case of children, the parents, gave their informed written consent to be included in the study. Samples were collected within $4 \mathrm{yr}$ of the affected twins or siblings meeting the established criteria for disease. Rheumatoid arthritis (RA) and dermatomyositis (DM) individuals, recruited at the National Institutes of Health (NIH) Clinical Center, respectively, fulfilled the Bohan and Peter and American College of Rheumatology criteria. For systemic lupus erythematosus (SLE) individuals, recruited at NIH Clinical Center and the Oklahoma Medical Research Foundation, the SLE Activity Index (SLEDAI) and Systemic Lupus International Collaborative Clinics/American College of Rheumatology (SLICC) Damage Index are shown (see Supplemental Table 4). For array-based DNA methylation profiling, 60 samples corresponding to 15 pairs of MZ twins discordant for disease plus two additional controls for each twin pair were used; specifically, these consisted of five pairs for each of the following diseases: SLE, RA, and DM. For each MZ twin pair, two additional unrelated age-, gender-, and race-matched healthy controls were used in arraybased DNA methylation profiling. For further DNA methylation analysis, 17 sibling pairs discordant for SLE were used: the above five $\mathrm{MZ}$ twin pairs plus one additional $\mathrm{MZ}$ twin pair, four $\mathrm{DZ}$ twin pairs, and seven non-twin sibling pairs. Also, a set of seven $M Z$ twins concordant for SLE was included. Homozygosity was determined by using highly polymorphic short tandem-repeat loci. White blood cells (WBCs) were extracted by standard procedures. WBC counts performed on an automatic Coulter counter were available for the majority of the samples. Total DNA was isolated from $\mathrm{WBCs}$ by standard procedures and stored at $-80^{\circ} \mathrm{C}$.

\section{Quantification of total DNA methylation}

$5 \mathrm{mC}$ content was quantified by high-performance capillary electrophoresis (HPCE), as previously described (Ballestar et al. 2003). In brief, immunoprecipitated DNA samples were speed-vac preconcentrated to $0.1 \mu \mathrm{g} / \mu \mathrm{L}$ and enzymatically hydrolyzed with nuclease $\mathrm{P} 1$ for $16 \mathrm{~h}$ at $37^{\circ} \mathrm{C}$, and subsequently treated with alkaline phosphatase (Sigma-Aldrich) for an additional $2 \mathrm{~h}$ at $37^{\circ} \mathrm{C}$. Samples were then directly injected in a Beckman MDQ HPCE apparatus. $5 \mathrm{mC}$ content was determined as the percentage of $5 \mathrm{mC}$ of total cytosines: $5 \mathrm{mC}$ peak area $\times 100 /(\mathrm{C}$ peak area $+5 \mathrm{mC}$ peak area).

\section{DNA methylation profiling using universal bead arrays}

A GoldenGate Methylation Cancer Panel I (Illumina) was used to quantify DNA methylation. The panel was developed to assay 1505 CpG sites selected from 807 genes, including oncogenes and tumor-suppressor genes, genes previously reported to be differentially methylated or differentially expressed, imprinted genes, genes involved in various signaling pathways, and genes responsible for DNA repair, cell cycle control, metastasis, differentiation, and apoptosis. For each CpG site, four probes were designed: two allele-specific oligos (ASOs) and two locus-specific oligos (LSOs). Each ASO-LSO oligo pair corresponded to either the methylated or unmethylated state of the CpG site. The reproducibility and validity of this array has been extensively validated (Bibikova et al. 2006). Bisulfite conversion of DNA samples was done using the EZ DNA methylation kit (Zymo Research). After this, the remaining steps were identical to those of the GoldenGate genotyping assay, using reagents and conditions supplied and recommended by Illumina. Two technical replicates of each bisulfite-converted sample were run. The results were all in close agreement and were averaged for subsequent analysis. The array was hybridized under a temperature gradient program, and arrays were imaged using a BeadArray Reader (Illumina). Image processing and intensity data extraction software and procedures were those described in Bibikova et al. (2006). Each methylation data point was represented as a combination of the Cy3 and Cy5 fluorescent intensities (Supplemental Data 1) from the M (methylated) and U (unmethylated) alleles (Supplemental Data 2). Background intensity computed from a set of negative controls was subtracted from each data point.

\section{Detection of differentially methylated genes from the methylation array and functional analysis}

A $t$-test was carried out as implemented in the GEPAS package (Montaner et al. 2006) to identify probes differentially methylated between SLE versus their respective healthy twins. $P$-values were corrected for multiple testing using the method proposed by Benjamini and Hochberg (1995) to control for the false discovery rate (FDR). Genes showing a $P$-value $<0.05$ (or FDR $<0.25$ ) and a minimum mean methylation change of $10 \%$ were considered differentially methylated. Gene Ontology analysis was done with the FatiGO tool (Al-Shahrour et al. 2004), which uses a statistical test (Fisher's exact test) to detect significant over-representation of GO terms in one of the sets (list of selected genes) with respect to the other one (the rest of the genome). Multiple test correction to account for the multiple hypothesis tested (one for each GO term) is applied to reduce false positives. GO terms with adjusted $P$-value $\leq$ 0.05 are considered significant. The relationship between the DNA methylation data (from standard bisulfite sequencing and/or quantitative bisulfite pyrosequencing) and the clinical data from the SLE individuals (activity and damage indexes), age and race were evaluated by the Pearson $\chi^{2}$-test. Multiple testing was performed for the SLE/Healthy MZ twin pair DNA methylation of resulting genes from the methylation array analysis and WBC counts.

\section{Amplification of unmethylated Alus (AUMA)}

DNA digestion with SmaI enzyme and ligation to the linker was performed as described (Rodriguez et al. 2008). The product was purified using the GFX Kit (Amersham Biosciences) and eluted in $250 \mu \mathrm{L}$ of sterile water. A chimeric primer comprised of the complementary linker sequence (ATTCGCAAAGCTCTGA), the cut SmaI site (GGG), and three additional nucleotides homologous to the Alu consensus sequence were used to enrich for $A l u$ sequences: AUMA-TTC (ATTCGCAAAGCTCTGAGGGTTC). In each PCR reaction only one primer was used at a time. Products were resolved on denaturing sequencing gels. Bands were visualized by silverstaining the gels. Faint bands with inconsistent display due to small variations in gel electrophoresis resolution were not considered. Band reproducibility was assessed with the analysis of PCR duplicates. AUMA fingerprints were visually checked for methylation differences between bands in different samples. Under these premises, a given band was scored according to three possible behaviors: hypomethylation (increased intensity), hypermethylation (decreased intensity), and no change (no substantial difference between samples). Only those bands showing clear 
changes in their intensities in the fingerprint were considered to represent methylation changes.

\section{Analysis of gene promoter methylation: Bisulfite sequencing and pyrosequencing}

The CpG island DNA methylation status was determined by sequencing bisulfite-modified genomic DNA. Bisulfite modification of genomic DNA was carried out as described by Herman et al. (1996). For each gene, primers were designed using the Methyl Primer Express v1.0 program (Applied Biosystems) corresponding to the region containing the oligonucleotide probe represented in the DNA methylation bead array. To validate the DNA methylation data obtained by conventional bisulfite genomic sequencing on a larger scale, bisulfite pyrosequencing (BPS) was performed according to standard protocols and evaluated with the Pyro Q-CpG 1.0.9 program (Biotage). Primer sequences, product lengths, and annealing temperatures used in the bisulfite sequencing and BPS PCR reactions are shown in Supplemental Table 5.

\section{Quantitative RT-PCR expression analyses}

We reverse-transcribed total RNA $(2 \mu \mathrm{g})$, treated with DNase (Ambion) using SuperScript II Reverse Transcriptase from Invitrogen. Quantitative real-time PCR (Q-RT-PCR) analysis was performed on an ABI 7900HT with Sybr green. Primer sequences are shown in Supplemental Table 5.

\section{Acknowledgments}

We thank Marina Corominas for technical assistance and Maria L. Ballestar for support in part of the analysis. We also thank the Lupus Association of Madrid (AMELYA). E.B. is supported by grants BFU2007-64216/BMC, CSD2006-49, and PI081346 (FIS) from the Spanish Ministry of Science and Innovation (MICINN). B.M.J. is funded by a BEFI Predoctoral Fellowship from the Carlos III Health Institute, Spain. This work was also supported in part by Intramural Research programs of the National Institute of Environmental Health Sciences, NIH and NIH grants (AR42460, AI31584, N01-AR62277, RR020143, AI24717, AR049084), the Alliance for Lupus Research, and the U.S. Department of Veterans Affairs.

\section{References}

Al-Shahrour F, Diaz-Uriarte R, Dopazo J. 2004. FatiGO: A web tool for finding significant associations of Gene Ontology terms with groups of genes. Bioinformatics 20: $578-580$

Ballestar E, Paz MF, Valle L, Wei S, Fraga MF, Espada J, Cigudosa JC, Huang TH, Esteller M. 2003. Methyl-CpG binding proteins identify novel site of epigenetic inactivation in human cancer. EMBO J 22: 6335-6345.

Benjamini Y, Hochberg Y. 1995. Controlling the false discovery rate: A practical and powerful approach to multiple testing. J R Stat Soc Ser B Methodol 57: 289-300.

Bennett L, Palucka AK, Arce E, Cantrell V, Borvak J, Banchereau J, Pascual V. 2003. Interferon and granulopoiesis signatures in systemic lupus erythematosus blood. J Exp Med 197: 711-723.

Bibikova M, Lin Z, Zhou L, Chudin E, Garcia EW, Wu B, Doucet D, Thomas NJ, Wang Y, Vollmer E, et al. 2006. High-throughput DNA methylation profiling using universal bead arrays. Genome Res 16: 383-393.

Chaussabel D, Quinn C, Shen J, Patel P, Glaser C, Baldwin N, Stichweh D, Blankenship D, Li L, Munagala I, et al. 2008. A modular analysis framework for blood genomics studies: Application to systemic lupus erythematosus. Immunity 29: $150-164$

Choubey D, Panchanathan R. 2008. Interferon-inducible Ifi200-family genes in systemic lupus erythematosus. Immunol Lett 119: 32-41.

Corvetta A, Della Bitta R, Luchetti MM, Pomponio G. 1991. 5-Methylcytosine content of DNA in blood, synovial mononuclear cells and synovial tissue from patients affected by autoimmune rheumatic diseases. J Chromatogr 566: 481-491.
Deng C, Kaplan MJ, Yang J, Ray D, Zhang Z, McCune WJ, Hanash SM, Richardson BC. 2001. Decreased Ras-mitogen-activated protein kinase signaling may cause DNA hypomethylation in T lymphocytes from lupus patients. Arthritis Rheum 44: 397-407.

Dodge JE, Okano M, Dick F, Tsujimoto N, Chen T, Wang S, Ueda Y, Dyson N, Li E. 2005. Inactivation of Dnmt3b in mouse embryonic fibroblasts results in DNA hypomethylation, chromosomal instability, and spontaneous immortalization. J Biol Chem 280: 17986-17991.

Duan B, Croker BP, Morel L. 2007. Lupus resistance is associated with marginal zone abnormalities in an NZM murine model. Lab Invest 87: 14-28.

Encinas JA, Kuchroo VK. 2000. Mapping and identification of autoimmunity genes. Curr Opin Immunol 12: 691-697.

Espada J, Ballestar E, Santoro R, Fraga MF, Villar-Garea A, Németh A, LopezSerra L, Ropero S, Aranda A, Orozco H, et al. 2007. Epigenetic disruption of ribosomal RNA genes and nucleolar architecture in DNA methyltransferase 1. Nucleic Acids Res 35: 2191-2198.

Esteller M. 2008. Epigenetics in cancer. N Engl J Med 358: 1148-1159.

Fraga MF, Ballestar E, Paz MF, Ropero S, Setien F, Ballestar ML, Heine-Suñer D, Cigudosa JC, Urioste M, Benitez J, et al. 2005. Epigenetic differences arise during the lifetime of monozygotic twins. Proc Natl Acad Sci 102: 10604-10609.

Funauchi M, Yoo BS, Nozaki Y, Sugiyama M, Ohno M, Kinoshita K, Kanamaru A. 2002. Dysregulation of the granulocyte-macrophage colony-stimulating factor receptor is one of the causes of defective expression of CD80 antigen in systemic lupus erythematosus. Lupus 11: 317-321.

Gibbons RJ, McDowell TL, Raman S, O'Rourke DM, Garrick D, Ayyub H, Higgs DR. 2000. Mutations in ATRX, encoding a SWI/SNF-like protein, cause diverse changes in the pattern of DNA methylation. Nat Genet 24: 368-371.

Gourley M, Miller FW. 2007. Mechanisms of disease: Environmental factors in the pathogenesis of rheumatic disease. Nat Clin Pract Rheumatol 3: 172-180.

Graham RR, Kyogoku C, Sigurdsson S, Vlasova IA, Davies LR, Baechler EC, Plenge RM, Koeuth T, Ortmann WA, Hom G. 2007. Three functional variants of IFN regulatory factor 5 (IRF5) define risk and protective haplotypes for human lupus. Proc Natl Acad Sci 104: 6758-6763.

Heijmans BT, Tobi EW, Stein AD, Putter H, Blauw GJ, Susser ES, Slagboom PE, Lumey LH. 2008. Persistent epigenetic differences associated with prenatal exposure to famine in humans. Proc Natl Acad Sci 105: 1704617049.

Herman JG, Graff JR, Myöhänen S, Nelkin BD, Baylin SB. 1996. Methylation-specific PCR: A novel PCR assay for methylation status of CpG islands. Proc Natl Acad Sci 93: 9821-9826.

Hrubec Z, Robinette CD. 1984. The study of human twins in medical research. $N$ Engl J Med 310: 435-441.

International Consortium for Systemic Lupus Erythematosus Genetics (SLEGEN). 2008. Genome-wide association scan in women with systemic lupus erythematosus identifies susceptibility variants in ITGAM, PXK, KIAA1542 and other loci. Nat Genet 40: 204-210.

Kaminsky ZA, Tang T, Wang SC, Ptak C, Oh GH, Wong AH, Feldcamp LA, Virtanen C, Halfvarson J, Tysk C, et al. 2009. DNA methylation profiles in monozygotic and dizygotic twins. Nat Genet 41: 240-245.

Kaplan MJ, Lu Q, Wu A, Attwood J, Richardson B. 2004. Demethylation of promoter regulatory elements contributes to perforin overexpression in $\mathrm{CD}^{+}$lupus T cells. J Immunol 172: 3652-3661.

Kim ST, Fields PE, Flavell RA. 2007. Demethylation of a specific hypersensitive site in the Th2 locus control region. Proc Natl Acad Sci 104: 17052-17057.

Kondo T, Bobek MP, Kuick R, Lamb B, Zhu X, Narayan A, Bourc'his D, ViegasPéquignot E, Ehrlich M, Hanash SM. 2000. Whole-genome methylation scan in ICF syndrome: Hypomethylation of non-satellite DNA repeats D4Z4 and NBL2. Hum Mol Genet 9: 597-604.

Lu Q, Kaplan M, Ray D, Ray D, Zacharek S, Gutsch D, Richardson B. 2002. Demethylation of ITGAL (CD11a) regulatory sequences in systemic lupus erythematosus. Arthritis Rheum 46: 1282-1291.

Lu Q, Wu A, Richardson BC. 2005. Demethylation of the same promoter sequence increases CD70 expression in lupus T cells and T cells treated with lupus-inducing drugs. J Immunol 174: 6212-6219.

Lu Q, Wu A, Tesmer L, Ray D, Yousif N, Richardson B. 2007. Demethylation of CD40LG on the inactive $\mathrm{X}$ in T cells from women with lupus. J Immunol 179: 6352-6358.

Mann MR, Lee SS, Doherty AS, Verona RI, Nolen LD, Schultz RM, Bartolomei MS. 2004. Selective loss of imprinting in the placenta following preimplantation development in culture. Development 131: 3727-3735.

Montaner D, Tárraga J, Huerta-Cepas J, Burguet J, Vaquerizas JM, Conde L, Minguez P, Vera J, Mukherjee S, Valls J. 2006. Next station in microarray data analysis: GEPAS. Nucleic Acids Res 34: W486-W491.

Moser KL, Kelly JA, Lessard CJ, Harley JB. 2009. Recent insights into the genetic basis of systemic lupus erythematosus. Genes Immun 10: 373-379.

\section{Genome Research}


Murayama A, Sakura K, Nakama M, Yasuzawa-Tanaka K, Fujita E, Tateishi Y, Wang Y, Ushijima T, Baba T, Shibuya K. 2006. A specific CpG site demethylation in the human interleukin 2 gene promoter is an epigenetic memory. EMBO J 25: 1081-1092.

Nance WE, ed. 1978. Twin research, Part C: Clinical studies. Alan R Liss, New York.

Pitashny M, Schwartz N, Qing X, Hojaili B, Aranow C, Mackay M, Putterman C. 2007. Urinary lipocalin-2 is associated with renal disease activity in human lupus nephritis. Arthritis Rheum 56: 1894-1903.

Rai K, Huggins IJ, James SR, Karpf AR, Jones DA, Cairns BR. 2009. DNA demethylation in zebrafish involves the coupling of a deaminase, a glycosylase, and gadd45. Cell 135: 1201-1212.

Richardson B. 1986. Effect of an inhibitor of DNA methylation on T cells. II. 5-Azacytidine induces self-reactivity in antigen-specific $\mathrm{T}^{+}{ }^{+}$cells. Hum Immunol 17: 456-470.

Richardson B, Scheinbart L, Strahler J, Gross L, Hanash S, Johnson M. 1990. Evidence for impaired T cell DNA methylation in systemic lupus erythematosus and rheumatoid arthritis. Arthritis Rheum 33: 16651673.

Rodriguez J, Vives L, Jordà M, Morales C, Muñoz M, Vendrell E, Peinado MA. 2008. Genome-wide tracking of unmethylated DNA Alu repeats in normal and cancer cells. Nucleic Acids Res 36: 770-784.
Salvador JM, Hollander MC, Nguyen AT, Kopp JB, Barisoni L, Moore JK, Ashwell JD, Fornace AJ Jr. 2002. Mice lacking the p53-effector gene Gadd45a develop a lupus-like syndrome. Immunity 16: 499_ 508.

Schwarting A, Wada T, Kinoshita K, Tesch G, Kelley VR. 1998. IFN- $\gamma$ signaling is essential for the initiation, acceleration, and destruction of autoimmune kidney disease in MRL-Fas ${ }^{l p r}$ mice. J Immunol 161: 494-503.

Schwartz N, Michaelson JS, Putterman C. 2007. Lipocalin-2, TWEAK, and other cytokines as urinary biomarkers for lupus nephritis. Ann NY Acad Sci 1109: 265-274.

van Overveld PG, Lemmers RJ, Sandkuijl LA, Enthoven L, Winokur ST, Bakels F, Padberg GW, van Ommen GJ, Frants RR, van der Maarel SM. 2003. Hypomethylation of D4Z4 in 4q-linked and non-4q-linked facioscapulohumeral muscular dystrophy. Nat Genet 5: 315-317.

Yung R, Ray D, Eisenbraun JK, Deng C, Attwood J, Eisenbraun MD, Johnson K, Miller RA, Hanash S, Richardson B. 2001. Unexpected effects of a heterozygous dnmt1 null mutation on age-dependent DNA hypomethylation and autoimmunity. J Gerontol A Biol Sci Med Sci 56: B268-B276.

Received September 4, 2009; accepted in revised form November 10, 2009. 


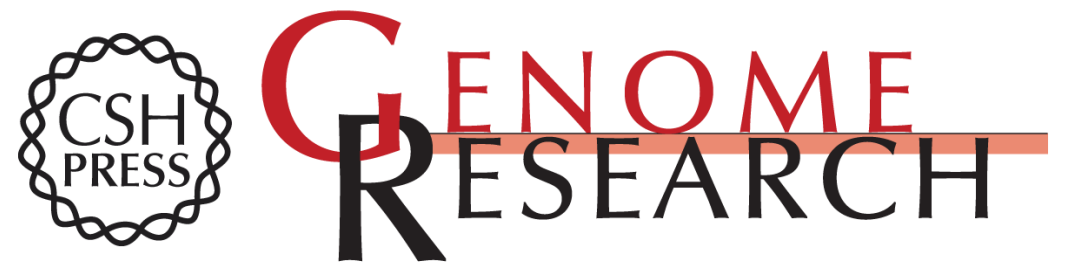

\section{Changes in the pattern of DNA methylation associate with twin discordance in systemic lupus erythematosus}

Biola M. Javierre, Agustin F. Fernandez, Julia Richter, et al.

Genome Res. 2010 20: 170-179 originally published online December 22, 2009

Access the most recent version at doi:10.1101/gr.100289.109

Supplemental Material

References

License

Email Alerting Service
http://genome.cshlp.org/content/suppl/2009/11/23/gr.100289.109.DC1

This article cites 44 articles, 14 of which can be accessed free at: http://genome.cshlp.org/content/20/2/170.full.html\#ref-list-1

Receive free email alerts when new articles cite this article - sign up in the box at the top right corner of the article or click here.

\section{Affordable, Accurate} Sequencing. 